\title{
Advances in Application of Fuzzy Sets in Electrical Engineering
}

\author{
Aditya Jain, Balakrushna Tripathy \\ School of Computing Science and Engineering VIT University, Vellore, India
}

\begin{tabular}{l} 
Article Info \\
\hline Article history: \\
Received Sep 13, 2017 \\
Revised Nov 15, 2017 \\
Accepted Nov 22, 2017 \\
\hline
\end{tabular}

\section{Keyword:}

Applications

Electrical

Fuzzy logic

Fuzzy sets

\begin{abstract}
Initially a theory, today fuzzy logic has become an operational technique. Used alongside other advanced control techniques, it is making a discrete but appreciated appearance in various electric systems. In the majority of present-day applications, fuzzy logic allows many kinds of designer and operator qualitative knowledge in electrical automation to be taken into account. Fuzzy logic began to interest the media at the beginning of the nineties. The numerous applications in electrical and electronic household appliances, particularly in Japan, were mainly responsible for such interest. Washing machines not requiring adjustment, camcorders with Steadyshot (TM) image stabilization and many other innovations brought the term "fuzzy logic" to the attention of a wide public.
\end{abstract}

Copyright $(0) 2017$ Institute of Advanced Engineering and Science. All rights reserved.

\section{Corresponding Author:}

Aditya Jain,

School of Computing Science and Engineering,

VIT University, Vellore, India-632014.

\section{THE HISTORY OF FUZZY SETS}

Fuzzy sets were acquainted by Zadeh in 1965 with speak to/control information and data having nonstatistical instabilities. It was particularly intended to scientifically speak to vulnerability and ambiguity and to give formalized instruments to managing the imprecision characteristic for some issues. Be that as it may, the account of fuzzy sets began considerably more before .To devise a brief hypothesis of sets, and later arithmetic, Aristotle set the purported "Laws of Thought".

One of these, the "Law of the Excluded Middle," expresses that each suggestion should either be True (T) or False (F). Notwithstanding when Parminedes proposed the principal variant of this law (around 400 Before Christ) there were solid and quick protests: for instance, Heraclitus suggested that things could be all the while True and not True.

\section{ORIGIN}

It was Plato who established the framework for what might get to be fuzzy sets, showing that there was a third area (past T and F) where these contrary energies "tumbled around." A methodical other option to the bi-esteemed sets of Aristotle was initially proposed by Łukasiewicz around 1920, when he depicted a three-esteemed sets, alongside the science to go with it. The third esteem he proposed can best be interpreted as the expression "conceivable," and he alloted it a numeric esteem amongst T and F. Inevitably, he proposed a whole documentation and proverbial framework from which he planned to infer present day science.

Later, he investigated four-esteemed setss, five-esteemed setss, and after that proclaimed that on a fundamental level there was nothing to keep the induction of a boundless esteemed logic. łukasiewicz felt that three-and vast esteemed setss were the most interesting, however he at last settled on a four-esteemed sets since it was by all accounts the most effortlessly versatile to Aristotelian sets.

It ought to be noticed that Knuth additionally proposed a three valued sets like Lukasiewicz's, from which he guessed that arithmetic would turn out to be considerably richer than in customary bi-esteemed sets. 
The thought of an unending esteemed sets was presented in Zadeh's fundamental work "Fuzzy Sets" where he depicted the arithmetic of fuzzy set hypothesis, and by expansion fuzzy sets. This hypothesis proposed making the enrollment work (or the qualities $\mathrm{F}$ and $\mathrm{T}$ ) work over the scope of genuine numbers [0, 1]. New operations for the math of sets were proposed, and appeared to be on a basic level no less than a speculation of great sets.

Fuzzy sets gives a surmising morphology that empowers rough human thinking capacities to be connected to learning based frameworks. The hypothesis of fuzzy sets gives a numerical quality to catch the instabilities connected with human intellectual procedures, for example, thinking and thinking.

The routine ways to deal with information representation do not have the methods for representating the significance of fuzzy ideas. As a result, the methodologies in light of first request sets and traditional likelihood hypothesis don't give a fitting applied structure to managing the representation of judicious information, since such learning is by its tendency both lexically loose and noncategorical. The advancement of fuzzy sets was persuaded in substantial measure by the requirement for a theoretical structure which can address the issue of vulnerability and lexical imprecision.

\section{FUZZY SET DEVELOPMENT IN ELECTRICAL ENGINEERING}

Today fuzzy sets has turned into an operational procedure. Utilized nearby other propelled control procedures, it is showing up in modern control computerization frameworks. Fuzzy sets does not really supplant routine control frameworks. Or maybe it finishes such frameworks. Its preferences originate from its capacity to:

a. formalize and reproduce the mastery of an administrator or originator in process control and tuning,

b. give a basic response to procedures which are hard to show,

c. persistently consider cases or special cases of various types, and continuously - join them into the skill,

d. consider a few factors and perform "weighted converging" of affecting into factors.

In the dominant part of present-day applications, fuzzy sets permits numerous sorts of architect and administrator subjective learning in framework mechanization to be considered. Fuzzy sets started to intrigue the media toward the start of the nineties. The various applications in electrical and electronic family apparatuses, especially in Japan, were mostly in charge of such intrigue. Clothes washers not requiring conformity, camcorders with Steadyshot (TM) picture adjustment and numerous different advancements brought the expression "fuzzy sets" to the consideration of a wide open.

In the auto business, programmed outfit changes, infusion and hostile to shake controls and ventilating can be upgraded because of fuzzy sets. In ceaseless and bunch generation forms, and in robotization frameworks (which is the subject of this Cahier Technique), applications have additionally expanded. Fuzzy sets has created around there as it is a basically even minded, successful and bland approach. It permits systematization of exact information and which is consequently difficult to control. The hypothesis of fuzzy sets offers a reasonable technique that is anything but difficult to actualize continuously applications, and empowers information of planners and administrators to be interpreted into element control frameworks.

This makes fuzzy sets ready to handle computerization of methodology, for example, startup and setting of parameters, for which few methodologies were beforehand accessible. This Cahier Technique portrays fuzzy sets and its application to generation forms.

\section{HISTORY AND CURRENT STATUS OF FUZZY SETS IN ELECTRICAL ENGINEERING}

Fuzzy sets was initially proposed by Lotfi A. Zadeh of the University of California at Berkeley in a 1965 paper. He expounded on his thoughts in a 1973 paper that presented the idea of "phonetic factors", which in this article compares to a variable characterized as a fuzzy set.[4] Other research took after, with the primary modern application, a concrete oven worked in Denmark, going ahead line in 1975.

Fuzzy frameworks were at first executed in Japan.

Enthusiasm for fuzzy frameworks was started by Seiji Yasunobu and Soji Miyamoto of Hitachi, who in 1985 gave reenactments that showed the attainability of fuzzy control frameworks for the Sendai railroad. Their thoughts were received, and fuzzy frameworks were utilized to control quickening, braking, and ceasing when the line opened in 1987.

In 1987, Takeshi Yamakawa showed the utilization of fuzzy control, through an arrangement of basic devoted fuzzy sets chips, in an "upset pendulum" test. This is a great control issue, in which a vehicle tries to keep a shaft mounted on its top by a pivot upright by moving forward and backward. Yamakawa along these lines made the exhibit more advanced by mounting a wine glass containing water and even a live mouse to the highest point of the pendulum: the framework kept up steadiness in both cases. Yamakawa in 
the long run went ahead to sort out his own fuzzy frameworks look into lab to adventure his licenses in the field.

Japanese designers thusly built up an extensive variety of fuzzy frameworks for both modern and purchaser applications. In 1988 Japan set up the Laboratory for International Fuzzy Engineering (LIFE), a helpful game plan between 48 organizations to seek after fuzzy research. The car organization Volkswagen was the main outside corporate individual from LIFE, dispatching a specialist for a length of three years.

Japanese buyer merchandise frequently consolidate fuzzy frameworks. Matsushita vacuum cleaners utilize microcontrollers running fuzzy calculations to cross examine tidy sensors and alter suction control appropriately. Hitachi clothes washers utilize fuzzy controllers to load-weight, texture blend, and soil sensors and naturally set the wash cycle for the best utilization of force, water, and cleanser.

Ordinance built up a self-adjusting camera that uses a charge-coupled gadget (CCD) to quantify the clarity of the picture in six districts of its field of view and utilize the data gave to figure out whether the picture is in core interest. It additionally tracks the rate of progress of focal point development amid centering, and controls its speed to avoid overshoot. The camera's fuzzy control framework utilizes 12 inputs: 6 to get the present clarity information gave by the CCD and 6 to gauge the rate of progress of focal point development. The yield is the position of the focal point. The fuzzy control framework utilizes 13 governs and requires 1.1 kilobytes of memory.

A mechanical ventilation system planned by Mitsubishi utilizes 25 warming tenets and 25 cooling rules. A temperature sensor gives contribution, with control yields sustained to an inverter, a compressor valve, and a fan engine. Contrasted with the past plan, the fuzzy controller warms and cools five times quicker, decreases control utilization by $24 \%$, builds temperature steadiness by a component of two, and utilizations less sensors.

Different applications researched or executed include: character and penmanship acknowledgment; optical fuzzy frameworks; robots, including one for making Japanese bloom courses of action; voicecontrolled robot helicopters (floating is an "exercise in careful control" fairly like the rearranged pendulum issue); recovery mechanical autonomy to give understanding particular arrangements (e.g. to control heart rate and pulse); control of stream of powders in film fabricate; lift frameworks; et cetera.

Take a shot at fuzzy frameworks is likewise continuing in the United State and Europe, despite the fact that on a less broad scale than in Japan. The US Environmental Protection Agency has researched fuzzy control for vitality proficient engines, and NASA has considered fuzzy control for computerized space docking: recreations demonstrate that a fuzzy control framework can enormously diminish fuel utilization. Firms, for example, Boeing, General Motors, Allen-Bradley, Chrysler, Eaton, and Whirlpool have taken a shot at fuzzy sets for use in low-control fridges, enhanced car transmissions, and vitality productive electric engines.

Maytag presented a "smart" dishwasher in view of a fuzzy controller and a "one-quit detecting module" that joins a thermistor, for temperature estimation; a conductivity sensor, to quantify cleanser level from the particles exhibit in the clothes washer; a turbidity sensor that measures scattered and transmitted light to gauge the ruining of the wash; and a magnetostrictive sensor to peruse turn rate. The framework decides the ideal wash cycle for any heap to get the best results with minimal measure of vitality, cleanser, and water. It even alters for dried-on sustenances by following the last time the entryway was opened, and evaluates the quantity of dishes by the quantity of times the entryway was opened.

Innovative work is additionally proceeding on fuzzy applications in programming, instead of firmware, plan, including fuzzy master frameworks and reconciliation of fuzzy sets with neural-system thus called versatile "hereditary" programming frameworks, with a definitive objective of building "self-learning" fuzzy control frameworks. These frameworks can be utilized to control mind boggling, nonlinear element plants, for instance, human body.

\section{THEORY OF FUZZY SETS}

\subsection{Notion of Incomplete Enrollment}

In the sets hypothesis, a component either has a place or does not have a place with a set. The thought of a set is utilized as a part of numerous scientific hypotheses. This key idea, be that as it may, does not consider circumstances which are yet both basic and regular. Discussing natural products, it is anything but difficult to characterize the arrangement of apples. Be that as it may, it is harder to characterize the arrangement of ready apples. We comprehend that an apple matures logically... the idea of a ready apple is in this manner a progressive one.

The idea of a fuzzy set was made keeping in mind the end goal to consider circumstances of this kind. The hypothesis of fuzzy sets depends on the thought of incomplete participation: every component has 
a place halfway or slowly to the fuzzy sets that have been characterized. The layouts of each fuzzy set (see Figure 1. are not "fresh", but rather "fuzzy" or "steady".

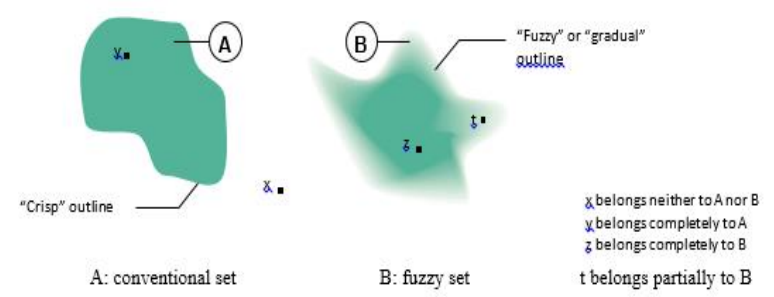

Figure 1. Comparison of a Conventional Set and a Fuzzy Set

\subsection{Membership functions}

A fuzzy set is defined by its "membership function" which corresponds to the notion of a "characteristic function" in classical logic. Let us assume that we want to define the set of people of "medium height". In classical logic, we would agree for example that people of medium height are those between 1.60 $\mathrm{m}$ and $1.80 \mathrm{~m}$ tall. The characteristic function of the set (see Figure 2 ) gives " 0 " for heights outside the range $[1.60 \mathrm{~m} ; 1.80 \mathrm{~m}]$ and " 1 " for heights in that range. The fuzzy set of people of "medium height" will be defined by a "membership function" which differs from a characteristic function in that it can assume any value in the range [0;1]. Each possible height will be assigned a "degree of membership" to the fuzzy set of "medium heights" (see Figure 3) between 0 and 1.

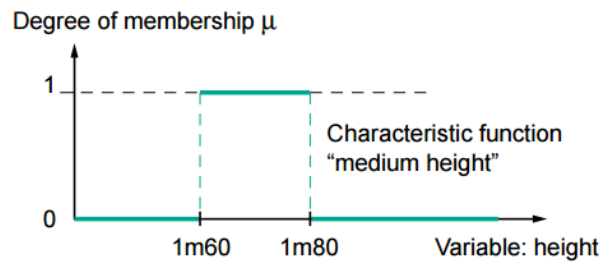

Fig. 2 : characteristic function.

Figure 2. Characteristic Function

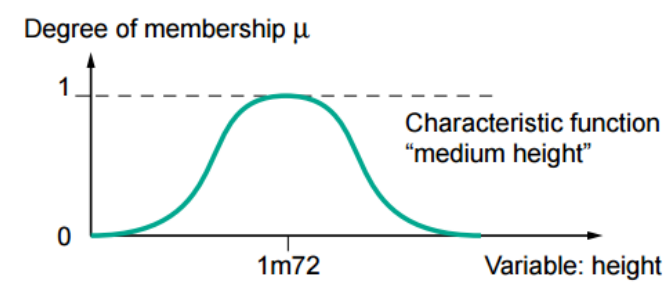

Fig. 3 : membership function.

Figure 3. Membership Funtion

A number of fuzzy sets can be defined on the same variable, for example the sets "small height", "medium height" and "tall height", each notion being explained by a membership function (see Figure 4).

This example shows the graduality that enables fuzzy logic to be introduced. A $1.80 \mathrm{~m}$ tall person belongs to the "tall" set with a degree of 0.3 , and to the set "medium height" with a degree of 0.7 . In classical logic, the change from average to tall would be sudden. A $1.80 \mathrm{~m}$ person would then be of medium height, whereas a $1.81 \mathrm{~m}$ person would be tall, an assertion which shocks intuition. The variable (for example: height) as well as the terms (for example: medium, tall) defined by the membership functions, are known as linguistic variable and linguistic term respectively. As we shall see further on, both linguistic variables and terms can be used directly in rules. Membership functions can assume any shape. However they are often defined by straight segments and said to be "piece-wise linear" (see Figure 5). "Piece-wise linear" membership functions are frequently used as:

a. They are straightforward,

b. They contain focuses permitting meaning of regions where the thought is valid and regions where it is false, consequently improving the social affair of skill.

We have utilized participation elements of this kind in whatever is left of this archive. At times, enrollment capacities might be equivalent to 1 for a solitary estimation of the variable, and equivalent to 0 somewhere else. They are then known as "singleton participation capacities". A fuzzy singleton (see Figure 6) characterized on a genuine variable (tallness) is the expression in the fuzzy field of a particular esteem (Paul's stature) of this variable. 


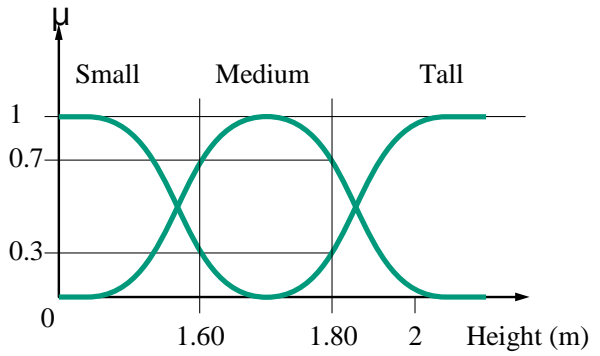

Figure 4. Membership Function, Variable and Linguistic Term

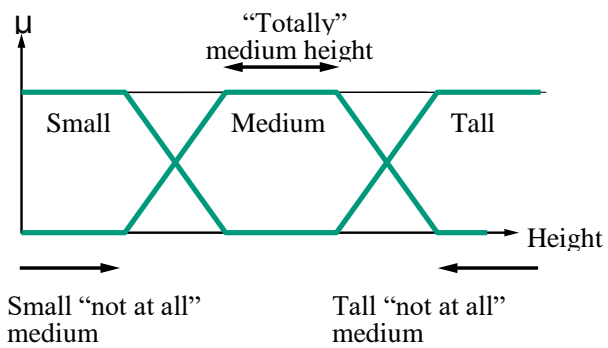

Figure 5. Piece-wise Direct Enrollment Capacities

Fuzzification - Degree of membership Fuzzification enables a real value to be converted into a fuzzy one. It consists of determining the degree of membership of a value (measured by example) to a fuzzy set. For example (see Figure 7), if the current value of the "input" variable is 2, the degree of membership to the "low input" membership function is equal to 0.4 which is the result of the fuzzification. We can also say that the "low input" proposal is true at 0.4. We then talk of degree of truth of the proposal. Degree of membership and degree of truth are therefore similar notions.

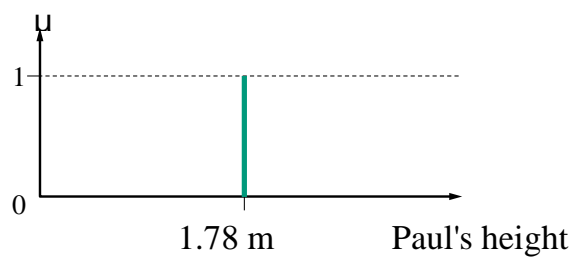

Figure 6. Singleton membership function

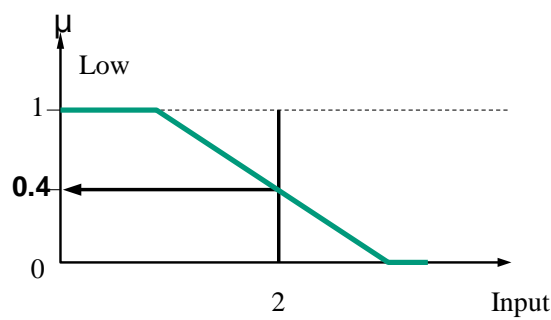

Figure 7. Fuzzification

\section{X,R MODELS, THEIR ANALYSIS, AND APPLICATIONS \\ 6.1. Substation Planning in Electrical Power Systems}

Among a group of force framework extension issues, the issue of substation arranging is of a key significance. It talks about how to consider the vulnerability figure the arrangement of this issue. The examination of a power utility gathering of substations is done to choose an answer among three options, considering their aggregate expenses with the instability of loan fees displayed as trapezoidal fuzzy numbers. This examination does not offer ground to continue with a persuading choice. One conceivable approach to beat this trouble is the utilization of the entrenched approach in basic leadership hone: the multi criteria investigation of choices in a fuzzy situation, when the use of extra criteria, including criteria of subjective character, serves as persuading intends to get the choice instability districts. The utilization of this approach is exemplified by examining a similar power utility gathering, with the utilization of the three fundamental systems for basic leadership depicted previously.

\subsection{Neighborhood Reactive Power Source Choice}

The issue of picking a neighborhood responsive power source at a power framework transport with receptive power lack on the premise of applying the criteria of unwavering quality, speculation level, and control rate. Its answer is connected with the development and examination of the $\mathrm{X}, \mathrm{R}$ models with fuzzy requesting of the considered criteria.

\subsection{Choice of Technology in Renewable Energy Diffusion Plan}

The issue of selecting the appropriated innovation in a renewable vitality dispersion get ready for the Sardinia area. This issue is determined on the premise of examining options with utilizing the idea of fuzzy lion's share. Specifically, the accompanying choices of vitality sources have been considered as 
choices: 1) sun oriented (residential sun powered water radiators), 2) (wind tur-bines of sort network associated), 3) pressure driven (hydro plants in induction plans), and 4) biomass (joined warmth and power plants bolstered by rural squanders or vitality crops).

The accompanying criteria have been considered: 1) focuses of essential vitality sparing in a local scale, 2) support capacity as indicated by Greenhouse poison discharges, 3) consistence of establishment and upkeep necessities with neighborhood specialized conditions, 4) congruity and consistency of exhibitions, 5) showcase development, and 6) similarity with political, authoritative, and managerial circumstance.

The initial two criteria are of a quantitative character. The third, fourth, and 6th criteria are of a subjective character and have been assessed through the arrangement of three fuzzy qualities. The fifth measure is additionally of a subjective character and has been assessed through the arrangement of five fuzzy qualities.

\subsection{Prioritization in Distribution System Maintenance Planning}

The issue of prioritization in support arranging goes for guaranteeing unwavering quality and administration nature of force supply through system and hardware preventive upkeep. CEMIG actualizes a methodology to understand the upkeep in dispersion frameworks as indicated by the arrangements proposed by makers of system components and gear. The prioritization methods permit one to consider parameters of system components and hardware, as well as elements identified with the states of

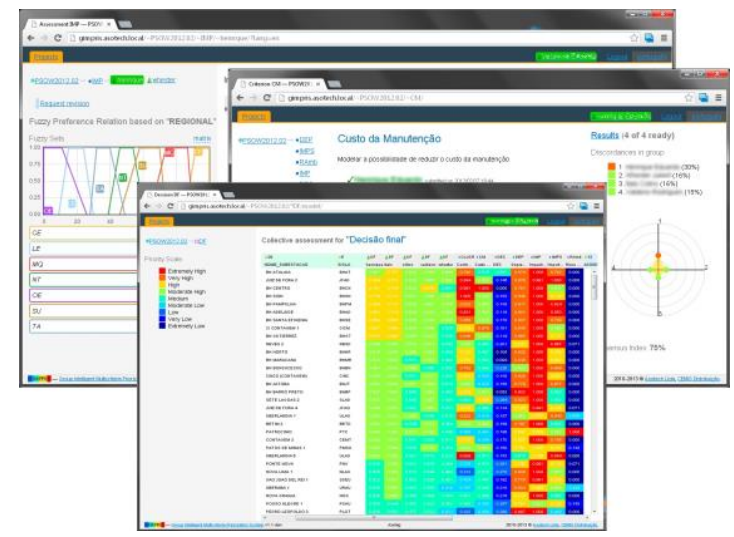

Figure 8. Electronic Cooperative Choice Making Environment for Prioritization in Dissemination Framework Support Arranging

Their operation not with standing the disappointment dangers, which can be surveyed through the measurable investigation, components connected with the effect of these disappointments are considered in the prioritization procedure. Among them it is conceivable to say quantitative components (number of purchasers, vitality requests, and so on.) and in addition subjective variables (political effect, upkeep intricacy, and so on.).

The registering framework for prioritization in upkeep arranging GIMPRIS executed for CEMIG ace vides collective choice making environment in the electronic stage (Figure 8). The adaptable apparatuses for inclination and total displaying are intended to amplify the abilities of X,R models for prioritization of more than 50,000 gear things. The dynamic gathering administration conspire actualized inside the framework al-lows a director to productively control the procedure of meeting to agreement, while directing up to 10 specialists all the while.

\section{AUTOMATED STOPPING DEVICES}

As a first case, consider a stopping automation, coordinated by a microcontroller chip as shown in Figure 9. The microcontroller needs to settle on choices in view of brake temperature, speed, and different factors in the framework. The variable "temperature" in this framework can be subdivided into a scope of "states": "icy", "cool", "direct", "warm", "hot", "and extremely hot". The move starting with one state then onto the next is difficult to characterize.

A self-assertive static edge may be set to partition "warm" from "hot". For instance, at precisely 90 degrees, warm finishes and hot starts. Be that as it may, this would bring about a spasmodic change when the 
info esteem disregarded that edge. The move wouldn't be smooth, as would be required in braking circumstances. The path around this is to make the states fuzzy. That is, permit them to change continuously starting with one state then onto the next. Keeping in mind the end goal to do this there must be a dynamic relationship set up between various components. We begin by characterizing the info temperature states utilizing "participation capacities":

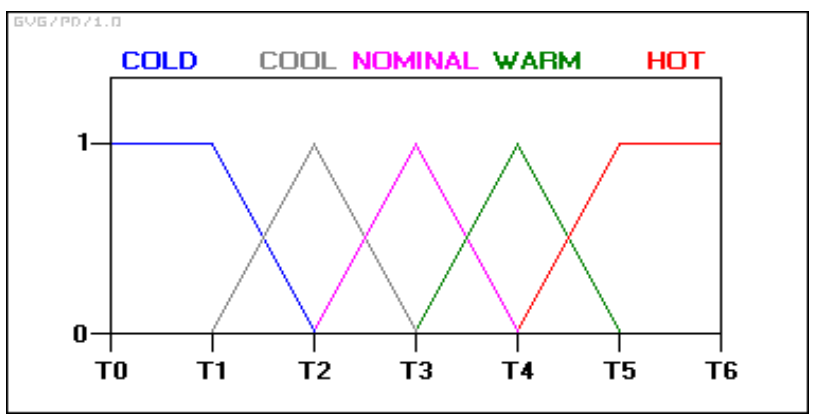

Figure 9. Coordinated by a Microcontroller Chip

With this plan, the info variable's express no longer hops unexpectedly starting with one state then onto the next. Rather, as the temperature transforms, it loses esteem in one enrollment work while picking up esteem in the following. At the end of the day, its positioning in the classification of icy declines as it turns out to be all the more very positioned in the hotter class.

At any examined time span, "reality esteem" of the brake temperature will quite often be in some degree a portion of two participation capacities: i.e.: '0.6 ostensible and 0.4 warm', or ' 0.7 ostensible and 0.3 cool', et cetera.

The above illustration shows a straightforward application, utilizing the reflection of qualities from various qualities. This exclusive speaks to one sort of information, in any case, for this situation, temperature. Adding extra refinement to this slowing mechanism, should be possible by extra elements, for example, footing, speed, inactivity, set up in element capacities, as per the outlined fuzzy system.

\section{LEGITIMATE ELUCIDATION OF FUZZY CONTROL}

Notwithstanding the appearance there are a few challenges to give a thorough consistent translation of the IF-THEN principles. For instance, decipher a govern as though (temperature is "frosty") THEN (radiator is "high") by the main request equation Cold(x) $\rightarrow \operatorname{High}(\mathrm{y})$ and expect that $\mathrm{r}$ is an information with the end goal that Cold(r) is false. At that point the equation Cold(r) $\rightarrow \operatorname{High}(\mathrm{t})$ is valid for any $\mathrm{t}$ and in this manner any $t$ gives a right control given r. A thorough consistent support of fuzzy control is given in Hájek's book (see Chapter 7) where fuzzy control is spoken to as a hypothesis of Hájek's essential logic.[2] Also in Gerla 2005 [9] another coherent way to deal with fuzzy control is proposed in light of fuzzy sets programming. Indeed, indicate by $f$ the fuzzy capacity emerging of an IF-THEN frameworks of principles. At that point we can make an interpretation of this framework into a fuzzy program $\mathrm{P}$ containing a progression of guidelines whose head is "Good(x,y)". The understanding of this predicate at all fuzzy Herbrand model of $\mathrm{P}$ concurs with $\mathrm{f}$. This gives facilitate valuable apparatuses to fuzzy control.

\section{CONCLUSION}

Initially a theory, today fuzzy logic has become an operational technique. Used alongside other advanced control techniques, it is making a discrete but appreciated appearance in industrial electric systems. In the majority of present-day applications, fuzzy logic allows many kinds of designer and operator qualitative knowledge in system automation to be taken into account. Fuzzy logic began to interest the media at the beginning of the nineties. The numerous applications in electrical and electronic household appliances, particularly in Japan, were mainly responsible for such interest. Washing machines not requiring adjustment, camcorders with Steadyshot (TM) image stabilization and many other innovations brought the term "fuzzy logic" to the attention of a wide public. 


\section{REFERENCES}

[1] Kevin M. Passino and Stephen Yurkovich, Fuzzy Control, Addison Wesley Longman, Menlo Park, CA, 1998 (522 Pages).

[2] Kazuo Tanaka; Hua O. Wang (2001). Fuzzy Control Systems Design and Analysis: A Linear Matrix Inequality Approach. John Wiley and Sons. ISBN 978-0-471-32324-2.

[3] Cox, E. (Oct. 1992). Fuzzy Fundamentals. Spectrum, IEEE, 29:10. Pp. 58-61.

[4] Cox, E. (Feb. 1993) Adaptive Fuzzy Systems. Spectrum, IEEE, 30:2. Pp. 7-31.

[5] Jan Jantzen, "Tuning Of Fuzzy PID Controllers", Technical University of Denmark, Report 98-H 871, September 30, 1998. [1].

[6] Jan Jantzen, Foundations of Fuzzy Control. Wiley, 2007 (209 Pages) (Table Of Contents)

[7] Computational Intelligence: A Methodological Introduction By Kruse, Borgelt, Klawonn, Moewes, Steinbrecher, Held, 2013, Springer, ISBN 9781447150121

[8] S. A. Orlovsky, "Problems of Decision Making With Fuzzy Information," Nauka, Moscow, 1981 (In Russian).

[9] C. R. Barrett, P. K. Patanalk And M Salles, "On Choosing Rationally When Preferences Are Fuzzy," Fuzzy Sets And Systems, Vol. 34, No. 2, 1990, Pp. 197-212. Doi:10.1016/0165-0114(90)90159-4.

[10] D. Bouyssou, "Acyclic Fuzzy Preference and the Or- Lovski Choice Function: A Note," Fuzzy Sets And Systems, Vol. 89, No. 1, 1997, Pp. 107-111. Doi:10.1016/S0165-0114(96)00078-4

[11] R. R. Yager, "On Ordered Weighted Averaging Aggrega- Tion Operators in Multi-Criteria Decision Making," IEEE Transactions on Systems, Man And Cybernetics, Vol. 18, No. 2, 1988, Pp. 183-190. Doi:10.1109/21.87068.

[12] W.-X, Li And B.-Y. Li, "An Extension of The Promethee II Method Based on Generalized Fuzzy Numbers," Expert Systems With Applications, Vol. 37, No. 7, Pp. 5314-5319.

[13] R. O. Parreiras, P. Ya. Ekel, J. S. C. Martini And R. M. Palhares, "A Flexible Consensus Scheme for Multicriteria Group Decision Making Under Linguistic Assessments,” Information Sciences, Vol. 180, No. 7, 2010, Pp. 1075 1089. Doi:10.1016/J.Ins.2009.11.046.

[14] R. O. Parreiras, P. Ya. Ekel And D. C. Morais, "Fuzzy Set Based Consensus Schemes for Multicriteria Group DeciSion Making Applied to Strategic Planning," Group Deci- Sion And Negotiation, Vol. 21, No. 2, 2012, Pp. 153183. Doi:10.1007/S10726-011-9231-0. 